\title{
Supraglotoplastia endoscópica em crianças com laringomalacia grave com e sem doença neurológica associada
}

\author{
Endoscopic supraglottoplasty in children with severe laryngomalacia \\ with and without neurological impairment
}

\author{
José C. Fraga ${ }^{1}$, Luciano Schopf ${ }^{2}$, Vanessa Volker ${ }^{3}$, Simone Canani ${ }^{4}$
}

\section{Resumo}

Objetivos: descrever as indicações e resultados da supraglotoplastia endoscópica em crianças com e sem lesão neurológica que apresentavam laringomalacia grave.

Métodos: estudo retrospectivo de 8 crianças com laringomalacia grave submetidas a supraglotoplastia endoscópica com material de microcirurgia. Quatro crianças tinham paralisia cerebral (todas masculinas, idade média 6 anos) e 4 eram crianças sem doença neurológica ( 3 femininas, idade média de 11,5 meses). As indicações da cirurgia foram dificuldade respiratória em repouso e/ou esforço, dificuldade de deglutição, deficiência de crescimento e baixa saturação transcutânea de oxigênio. Estudo polissonográfico realizado nas últimas duas crianças mostrou dessaturação na oximetria, apnéia e hipoventilação durante o sono. As crianças receberam corticóide e antibiótico no pré-operatório.

Resultados: todas as crianças sem doença neurológica apresentaram melhora significativa do estridor e da obstrução ventilatória. Das crianças com paralisia cerebral, uma necessitou traqueostomia logo após a cirurgia por edema e tecido de granulação supraglótico. As outras três apresentaram melhora clínica inicial, mas pioraram progressivamente, apesar da realização de outros procedimentos: uma necessitou nova supraglotoplastia após 6 meses; outra necessitou traqueostomia após 7 meses. Aquelas sem traqueostomia persistem com obstrução ventilatória grave. Não foi observada complicação do procedimento cirúrgico.

Conclusões: 1) a supraglotoplastia é bem tolerada e sem complicações na criança; 2) a supraglotoplastia, neste estudo, mostrouse eficaz no tratamento da laringomalacia grave em crianças sem paralisia cerebral; entretanto, a supraglotoplastia não melhorou a obstrução ventilatória de crianças com paralisia cerebral e laringomalacia grave.

J Pediatr (Rio J) 2001; 77 (5): 420-4: laringomalacia, supraglotoplastia endoscópica.

\begin{abstract}
Objective: to describe indications and results of supraglottoplasty for severe laryngomalacia in children with or without neurological impairment.

Methods: eight children with severe laryngomalacia submitted to endoscopic supraglottoplasty were retrospectively studied. Four had neurological impairment (male, mean age 6 years), and 4 did not present neurological problems (3 female, mean age 11.5 months). Surgery indications were respiratory distress, feeding difficulties, failure to thrive, and low oxygen saturation. Polysomnographic evaluation was carried out on the last 2 children, showing abnormal oxygen saturation, obstructive apnea, and hypoventilation. All children received preoperative antibiotics and corticosteroids.

Results: all children without neurological impairment had significant relief of symptoms. Children with neurological impairment had different outcome: one needed tracheotomy immediately after surgery due to edema and supraglottic granulation tissue. The other three children presented initial relief of symptoms, but subsequent follow-up showed progressive airway obstruction: one needed another endoscopic surgery 6 months later; other needed tracheotomy 7 months later. The children who were not submitted to tracheostomy presented persistent severe airway obstruction. No endoscopic surgery complication was observed.
\end{abstract}

Conclusions: 1) Endoscopic supraglottoplasty is well tolerated and does not present complications when used in children; 2) Endoscopic supraglottoplasty was efficient in the treatment of children with severe laryngomalacia and in without neurological impairment; however, supraglottoplasty did not resolve airway obstruction in children with neurological impairment.

J Pediatr (Rio J) 2001; 77 (5): 420-4: laryngomalacia, endoscopic supraglottoplasty.

1. Professor Adjunto de Cirurgia Pediátrica da Faculdade de Medicina e do Curso de Pós-Graduação em Medicina: Cirurgia - UFRGS. Mestre e Doutor em Medicina. Cirurgião Pediátrico dos Hospitais de Clínicas e Moinhos de Vento - Porto Alegre, RS, Brasil.

2. Cirurgião Pediátrico. Aluno do Curso de Pós-graduação em Medicina: Cirurgia da UFRGS, nível Mestrado.

3. Aluna de Graduação da Faculdade de Medicina da UFRGS.

4. Pneumologista Pediátrica dos Hospitais de Clínicas e Moinhos de Vento. Médica do Laboratório do Sono do Hospital de Clínicas, Porto Alegre, RS, Brasil. Trabalho realizado nos Hospitais de Clínicas e Moinhos de Vento, Porto Alegre, RS, Brasil, e apresentado no XXI Congresso de Cirurgia Pediátrica realizado em Porto Alegre, de 22 a 26 de outubro de 2000. 


\section{Introdução}

A laringomalacia é definida como o distúrbio caracterizado pelo colapso das cartilagens laríngeas durante a inspiração, com obstrução da glote. Ela é a anomalia mais comum do laringe, bem como a causa mais freqüente de estridor na criança. Grandes séries de pacientes com anormalidades do laringe têm mostrado que a laringomalacia representa 50 a $75 \%$ dos diagnósticos ${ }^{1,2}$.

A manifestação característica da laringomalacia é o estridor inspiratório intermitente. $\mathrm{O}$ estridor usualmente aparece nas duas primeiras semanas de vida, é exacerbado pelo choro, agitação ou exercício, e melhora com a extensão do pescoço. O diagnóstico definitivo é realizado através de endoscopia respiratória, com a criança em respiração espontânea. $\mathrm{O}$ exame demonstra a presença e também a gravidade da obstrução ocasionada pelo colapso das cartilagens laríngeas.

$\mathrm{Na}$ maioria das crianças com laringomalacia, o prognóstico é favorável, já que a obstrução ventilatória não é intensa, e ocorre a melhora significativa dos sintomas antes dos 2 anos de idade. Entretanto, em cerca de $10 \%$ dos pacientes, a obstrução ventilatória é grave e ocasiona uma série de complicações: apnéia com risco de vida, cianose, insuficiência ventilatória, dificuldade de alimentação e de ganho de peso, e cor pulmonale. Até há alguns anos, a traqueostomia era indicada nestes pacientes ${ }^{3}$; contudo, devido às complicações da traqueostomia na criança ${ }^{4}$, a ressecção endoscópica do tecido supraglótico redundante (supraglotoplastia, epiglotoplastia ou ariepiglotoplastia) tornou-se a primeira opção no tratamento de crianças com laringomalacia grave ${ }^{3,5}$.

Em algumas crianças com paralisia cerebral, há o desenvolvimento de estridor inspiratório muito similar àquele visto na laringomalacia do lactente. Achados endoscópicos nestes pacientes também mostram a presença de mucosa redundante de aritenóides, encurtamento do ligamento ariepiglótico e flacidez da epiglote, muito semelhantes àqueles observados na laringomalacia. Este tipo de laringomalacia é denominado laringomalacia neurológica e, provavelmente, é resultante de algum distúrbio na inervação da musculatura do faringe e laringe. A cirurgia endoscópica também tem sido realizada nestes pacientes, com bons resultados 6 .

Este estudo tem o objetivo de avaliar, retrospectivamente, os resultados da supraglotoplastia (SGP) endoscópica em crianças com e sem paralisia cerebral que apresentavam laringomalacia grave.

\section{Métodos}

Foi realizado estudo retrospectivo de prontuários de 8 crianças submetidas a supraglotoplastia endoscópica para laringomalacia grave no Hospital Moinhos de Vento e no Hospital de Clínicas de Porto Alegre, no período de janeiro de 1995 a fevereiro de 2000. Havia 4 crianças com paralisia cerebral e 4 sem doença neurológica (Tabela 1). A média de idade no grupo com doença neurológica era 6 anos, e a mediana 8 anos e 7 meses; no grupo sem doença neurológica a média foi de 11,5 meses e a mediana, de 10,5 meses. Os pacientes foram considerados candidatos a cirurgia endoscópica devido a dificuldade respiratória em repouso e/ou esforço, dificuldade de deglutição, deficiência de crescimento e baixa saturação transcutânea de oxigênio. A dificuldade respiratória foi considerada grave quando provocava tiragem intercostal e esternal, cianose e dificuldade de alimentação, com engasgos freqüentes e ganho inadequado de peso. As duas últimas crianças operadas também realizaram polissonografia, que demonstrou vários episódios de dessaturação na oximetria, bem como apnéia e hipoventilação grave durante o sono. Dependendo da suspeita clínica, algumas crianças também realizaram radiografia de tórax, pesquisa de refluxo gastroesofágico e ecocardiograma.

O diagnóstico de laringomalacia foi feito através de broncoscopia, realizada sob anestesia geral, com a criança em respiração espontânea. Mesmo após confirmado o diagnóstico da laringomalacia, realizava-se exame endoscópico completo da via aérea para excluir malformações associadas. No momento da cirurgia, realizava-se laringoscopia para expor a laringe, e se introduziam instrumentos de microcirurgia (tesoura e pinça) através da boca, com secção e remoção do tecido supraglótico redundante ou de porção lateral da epiglote (Figura 1). O tipo de cirurgia endoscópica dependeu do tipo de obstrução que cada criança apresentava (Figura 1). Todos os pacientes receberam, no préoperatório, corticóide (dexametasona) e antibiótico profilático (cefalotina), que eram mantidos por 48 horas.

Se possível, as crianças não eram intubadas durante ou após o procedimento cirúrgico. No pós-operatório, elas eram observadas na Unidade de Tratamento Intensivo Pe-

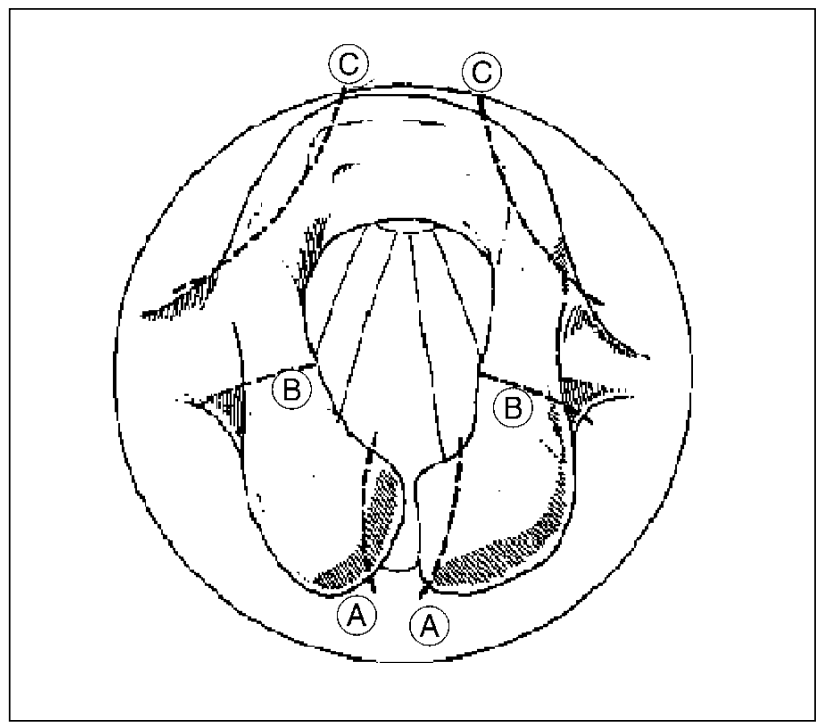

Figura 1 - Tipos de cirurgia endoscópica em crianças com laringomalacia grave: A) excisão da mucosa redundante da aritenóide; B) secção do ligamento ariepiglótico; C) remoção da porção lateral da epiglote 
diátrico e recebiam nebulização com adrenalina, se apresentassem qualquer dificuldade ventilatória.

\section{Resultados}

Todas as crianças deste estudo apresentavam estridor e dificuldade respiratória grave, que foram responsáveis por vários episódios de hospitalização e/ou atendimento em emergência.

Todas as crianças com doença neurológica apresentavam paralisia cerebral e eram do sexo masculino (Tabela 1). Uma criança apresentava Síndrome de Cornelio de Lange, e duas tinham refluxo gastroesofágico controlados com medicação. Os achados endoscópicos observados nestas crianças foram colapso bilateral de aritenóides (100\%) e de epiglote (25\%), e as indicações de cirurgia foram dificuldade respiratória em repouso $(75 \%)$ e baixa saturação de oxigênio na monitorização transcutânea (50\%).

Das crianças sem doença neurológica, 3 eram do sexo feminino e 1 do sexo masculino (Tabela 1). Nenhuma apresentava outra doença associada. Os achados endoscópicos foram os mesmos observados no grupo anterior, e as indicações de cirurgia foram dificuldade respiratória em repouso e/ou esforço (100\%), deficiência de crescimento $(50 \%)$ e dificuldade deglutição $(25 \%)$. As duas últimas crianças operadas também tiveram estudo polissonográfico alterado.

O tipo de cirurgia endoscópica foi o mesmo em ambos os grupos (Tabela 2). Os pacientes com doença neurológica necessitaram intubação traqueal logo após o procedimento. Uma criança teve melhora significativa da disfunção venti- latória após a cirurgia, apresentando apenas estridor leve ocasional. Entretanto, o estridor e a disfunção ventilatória pioraram gradativamente, tendo sido necessária a realização de traqueostomia 7 meses após a cirurgia endoscópica. Outra criança apresentou edema subglótico e formação de tecido de granulação supraglótico, que impossibilitou a extubação, tendo sido realizada traqueostomia 6 dias após a cirurgia endoscópica. Outra criança apresentou melhora inicial, mas houve recorrência gradativa da obstrução respiratória, tendo sido necessária nova cirurgia endoscópica 6 meses após a cirurgia prévia. Na cirurgia, foi ressecado novamente o tecido redundante da aritenóide direita. A criança evoluiu bem, inicialmente, mas no seguimento tardio persiste com estridor e disfunção ventilatória intermitente. A última criança operada apresentou melhora inicial da obstrução ventilatória, mas persiste com estridor leve intercalado com piora importante da obstrução, com várias internações e consultas na emergência devido à disfunção ventilatória grave. O tempo médio de seguimento destas crianças é de 2 anos e 1 mês.

A evolução das crianças com laringomalacia grave e sem doença neurológica pode ser observada na Tabela 2. Não houve necessidade de intubação traqueal logo após a cirurgia, apesar de todas terem sido submetidas a nebulizações freqüentes com adrenalina no pós-operatório imediato, devido à disfunção ventilatória, que piorou logo após a cirurgia. Houve melhora completa da obstrução ventilatória em todas as crianças, apesar da maioria delas apresentar estridor leve intermitente. Também ocorreu redução significativa das internações e consultas na emergência por disfunção ventilatória alta. O seguimento destas crianças é em média de 1 ano e 11 meses.

Tabela 1 - Características das crianças com laringomalacia grave com e sem doença neurológica, submetidas a supraglotoplastia endoscópica

\begin{tabular}{|c|c|c|c|c|}
\hline \multirow[b]{2}{*}{ Sexo } & \multicolumn{2}{|l|}{ Doença Neurológica $(n=4)$} & \multicolumn{2}{|c|}{ Ausência de Doença Neurológica $(n=4)$} \\
\hline & Masc - 4 & & Masc - 1; Fem - 3 & \\
\hline $\begin{array}{l}\text { Idade cirurgia } \\
\text { - média (variação) } \\
\text { - mediana }\end{array}$ & $\begin{array}{l}6 \text { anos }(5 a 1 m-13 a) \\
8 \text { anos } 7 \text { meses }\end{array}$ & & $\begin{array}{l}11,5 \text { meses }(1 \mathrm{~m}-1 \mathrm{a} 8 \mathrm{~m}) \\
10,5 \text { meses }\end{array}$ & \\
\hline Malformação associada & $\begin{array}{l}\text { RGE } \\
\text { Sind. Cornelio de Lange }\end{array}$ & $\begin{array}{l}-2 \\
-1\end{array}$ & Nenhuma & \\
\hline Indicação de cirurgia & $\begin{array}{l}\text { Dificuldade respiratória em repouso } \\
\text { Oximetria anormal }\end{array}$ & $\begin{array}{l}-3 \\
-2\end{array}$ & $\begin{array}{l}\text { Dificuldade respiratória } \\
\text { em repouso e/ou esforço } \\
\text { Deficiência de crescimento } \\
\text { Polissonografia anormal * } \\
\text { Dificuldade deglutição }\end{array}$ & $\begin{array}{l}-4 \\
-2 \\
-2 \\
-1\end{array}$ \\
\hline Achado endoscópico & $\begin{array}{l}\text { Colapso aritenóides bilateral } \\
\text { Colapso epiglote }\end{array}$ & $\begin{array}{l}-4 \\
-1\end{array}$ & $\begin{array}{l}\text { Colapso aritenóides bilateral } \\
\text { Colapso epiglote }\end{array}$ & $\begin{array}{l}-4 \\
-1\end{array}$ \\
\hline
\end{tabular}

* Oximetria anormal, apnéia grave e hipoventilação durante o sono. 
Tabela 2 - Tipo e resultado de cirurgia endoscópica em crianças com laringomalacia grave, com e sem doença neurológica

\begin{tabular}{|c|c|c|c|c|}
\hline & Doença Neurológica $(n=4)$ & & Ausência de Doença Neurológic & =4) \\
\hline Tipo de cirurgia & $\begin{array}{l}\text { - Ressecção unilateral aritenóide } \\
\text { - Ressecção bilateral aritenóides } \\
\text { - Epiglotectomia parcial }\end{array}$ & $\begin{array}{l}-2 \\
-2 \\
-1\end{array}$ & $\begin{array}{l}\text { - Ressecção unilateral aritenóide } \\
\text { - Ressecção bilateral aritenóides } \\
\text { - Epiglotectomia parcial }\end{array}$ & $\begin{array}{l}-2 \\
-2 \\
-1\end{array}$ \\
\hline $\begin{array}{l}\text { Necessidade intubação } \\
\text { após a cirurgia }\end{array}$ & - Todos (média de 3,2 dias) & & - Nenhum & \\
\hline Resultado inicial & $\begin{array}{l}\text { - Melhora inicial da obstrução } \\
\text { - Estridor intermitente } \\
\text { - Impossibilidade extubação } \\
\text { (traqueostomia) }\end{array}$ & $\begin{array}{l}-3 \\
-3 \\
-1\end{array}$ & $\begin{array}{l}\text { - Melhora completa da obstrução } \\
\text { - Estridor intermitente }\end{array}$ & $\begin{array}{l}-4 \\
-3\end{array}$ \\
\hline Necessidade de reoperação & $\begin{array}{l}\text { - Nova ressecção aritenóide } \\
\text { unilateral (6m após) }\end{array}$ & -1 & - Nenhum & \\
\hline Seguimento (tempo médio) & $\begin{array}{l}\text { - Piora progressiva da obstrução } \\
\text { (uma traqueostomizada após } 7 \mathrm{~m}) \\
\text { - Manutenção de traqueostomia prévia } \\
\text { ( } 2 \text { anos } 1 \text { mês) }\end{array}$ & $\begin{array}{l}-3 \\
-1\end{array}$ & $\begin{array}{l}\text { - Estridor intermitente } \\
\text { (1 ano } 11 \text { meses) }\end{array}$ & -3 \\
\hline
\end{tabular}

\section{Discussão}

A laringomalacia geralmente manifesta-se alguns dias após o nascimento, através do estridor inspiratório. Nas crianças com paralisia cerebral, o estridor inicia em uma fase mais tardia, geralmente no final da infância. Isso explica a diferença de idade observada entre as crianças, com e sem doença neurológica, relatadas neste estudo.

A identificação de doenças ou malformações associadas é uma etapa importante no tratamento de crianças com laringomalacia grave. Alterações de traquéia ou brônquios devem ser excluídas pela endoscopia, pois alterações mais distais da via aérea precisam geralmente ser tratadas primeiro. Isso é importante no momento de realização da endoscopia, pois sempre se deve realizar o exame de toda a via aérea, mesmo após estabelecido o diagnóstico de laringomalacia grave. O diagnóstico e tratamento do refluxo gastroesofágico concomitante também é importante nestas crianças com laringomalacia grave, pois ele pode agravar a dificuldade respiratória ocasionada pela malacia. Assim, antes de se classificar a severidade da laringomalacia, é necessário o controle do refluxo gastroesofágico ${ }^{7}$.

A maioria das crianças com laringomalacia não necessita qualquer tipo de intervenção cirúrgica, pois há melhora da flacidez das cartilagens supraglóticas com o crescimento, ocorrendo o desaparecimento completo dos sintomas até os dois anos de idade ${ }^{1,2}$. Isso também é observado em crianças com paralisia cerebral e laringomalacia, visto que somente a minoria destes pacientes desenvolverá quadros graves de obstrução ventilatória, com necessidade de tratamento $^{6}$. As crianças com laringomalacia grave, definidas como aquelas que apresentam dispnéia obstrutiva, cianose, dificuldade de ganho de peso e cor pulmonale necessitam de tratamento cirúrgico 5 . É importante salientar que a severidade da laringomalacia não depende da intensidade do estridor, mas sim das manifestações decorrentes da obstrução ventilatória ${ }^{7}$.

A traqueostomia era tradicionalmente utilizada em crianças com laringomalacia grave. Na última década, a redução endoscópica do tecido supraglótico tem sido relatada como um método efetivo no tratamento destas crianças. Iglauer, em 1922, foi o pioneiro na realização da cirurgia endoscópica para o tratamento de estridor, ao realizar epiglotectomia ${ }^{8}$. Em 1928, Hasslinger relatou excelentes resultados na ressecção das pregas ariepiglóticas de três pacientes com laringomalacia ${ }^{9}$. Schwartz, em 1944, 10 propôs que a laringomalacia grave poderia ser tratada através da ressecção cirúrgica dos bordos laterais da epiglote. Entretanto, foi somente em 1984 que Lane et al. ${ }^{11}$, pela primeira vez, propuseram que a laringomalacia grave deveria ser tratada através da ressecção endoscópica da mucosa redundante de uma ou de ambas as aritenóides, e de ressecção das bordas laterais da epiglote. Desde então, vários trabalhos têm mostrado bons resultados com esta técni$\mathrm{ca}^{12-15}$.

Como observado em nosso estudo, a indicação mais comum de cirurgia para laringomalacia é a dificuldade ventilatória grave ocasionada pelo colapso das estruturas supraglóticas. Alguns autores têm relatado que alterações na polissonografia podem ser úteis na avaliação da gravidade da obstrução ventilatória. De acordo com Zarzal et al. ${ }^{12}$, 
em uma série de dez crianças operadas, 8 apresentavam apnéia obstrutiva durante o sono. No relato de 13 crianças, Holinger e Konior ${ }^{15}$ descreveram 8 crianças com apnéia durante o sono e 5 com episódios repetitivos de cianose. A utilização da polissonografia nas nossas últimas duas crianças operadas mostrou que este exame é extremamente útil para a indicação da cirurgia, pois ele quantifica a gravidade da obstrução ventilatória. A indicação de cirurgia passou a ser baseada não somente em dados subjetivos, mas também em resultados numéricos que avaliam a gravidade da obstrução.

A ressecção endoscópica do tecido supraglótico redundante é uma técnica cirúrgica simples, que foi bem tolerada por nossos pacientes. A melhora da ventilação já foi observada logo após o procedimento cirúrgico. A cirurgia endoscópica pode ser realizada com instrumento microcirúrgico ou com laser. Com a microcirurgia podem ocorrer pequenos sangramentos locais, que em geral cessam espontaneamente. Apesar de que a utilização do laser não provoca risco de sangramento, seu uso excessivo pode ocasionar edema e tecido de granulação local, com dificuldade de deglutição ou mesmo necessidade de intubação pós-operatória ${ }^{7}$. Independente do equipamento utilizado, o importante é a remoção mais conservadora possível de tecido supraglótico, retirando-se somente o mínimo necessário para desobstruir a entrada da glote. Dependendo da causa da obstrução ventilatória supraglótica, pode-se realizar um ou mais dos procedimentos cirúrgicos endoscópicos (remoção de um ou de ambos os tecidos aritenóides redundantes, secção dos ligamentos ariepiglóticos, ou retirada das bordas laterais da epiglote - Figura 1). Entretanto, é preferível realizar uma nova cirurgia endoscópica posteriormente, para remover um pouco mais de tecido, do que ressecar demasiadamente na primeira intervenção e ocasionar complicações, que em geral são de difícil tratamento ${ }^{3}$.

Os resultados obtidos com a supraglotoplastia para laringomalacia grave foram diferentes em crianças com e sem doença neurológica. As crianças sem paralisia cerebral apresentaram excelente resultado no acompanhamento inicial e tardio. Todas tiveram melhora da ventilação, apesar de apresentarem estridor leve ocasional. A melhora clínica destas crianças foi nítida, já que elas não necessitaram mais de internações ou consultas na emergência devido à dificuldade ventilatória.

As crianças com paralisia cerebral e laringomalacia grave tiveram resultado insatisfatório com a supraglotoplastia endoscópica, já que duas necessitaram traqueostomia e outras duas continuaram com disfunção ventilatória grave no seguimento a longo prazo. Estes resultados observados em nosso estudo são diferentes daqueles relatados por Hiu et al. ${ }^{6}$, que descreveram bom resultado com o uso desta técnica em 4 pacientes com paralisia cerebral e laringomalacia grave. Entretanto, neste último estudo, não há relato de tempo de seguimento das crianças. Nosso estudo mostrou que estes pacientes apresentam uma melhora sintomática inicial, mas a dificuldade ventilatória reapa- rece futuramente. Talvez isso seja decorrente da progressão do distúrbio neurológico da musculatura da faringe e laringe, com piora gradativa da disfunção ventilatória ${ }^{6}$.

Neste estudo não se observou nenhuma complicação da supraglotoplastia endoscópica. As complicações descritas com esta cirurgia são sangramento local, infecção e sepses, sinéquia interaritenóide, estenose supraglótica e aspiração ${ }^{5}$.

Em conclusão, nosso estudo mostrou que a supraglotoplastia é um procedimento seguro na criança, provocando melhora significativa da laringomalacia grave de crianças sem anormalidade neurológica. Crianças com paralisia cerebral não apresentaram melhora importante com a cirurgia. A necessidade do estudo de maior número de pacientes é necessária para uma conclusão definitiva sobre a utilidade desta cirurgia em crianças com laringomalacia grave.

\section{Referências bibliográficas}

1. Fraga JC, Nogueira A, Palombini B. Laringomalacia em criança Revisão de 92 casos. Revista AMRIGS 1993; 37:145-8.

2. Sichel JY, Dangoor E, Eliashar R, Halperin D. Management of congenital laryngeal malformation. Am J Otolaryngol 2000;21:22-30.

3. Wiatrak BJ. Congenital anomalies of the larynx and trachea. In: Jong AL, Kuppersmith RB. Update on the pediatric airway. Otolaryngol Clin North Am 2000; 33:91-110.

4. Gross CW. Medical management, nasotracheal intubation, and tracheostomy in the treatment of upper airway obstruction in children. Otolaryngol Clin North Am 1977; 10:157-66.

5. Kelly SM, Gray SD. Unilateral endoscopic supraglottoplasty for severe laryngomalacia. Arch Otolaryngol Head Neck Surg 1995; 121:1351-4.

6. Hui Y, Gaffney R, Crysdale WS. Laser aryepiglottoplasty for the treatment of neurasthenic laryngomalacia in cerebral palsy. Ann Otol Rhinol Laryngol 1995;104:423-6.

7. Roger G, Denoyelle F, Triglia JM, Garabedian EN. Severe laryngomalacia: surgical indications and results in 115 patients. Laryngoscope 1995;105:1111-7.

8. Narcy P, Andrieu-Guitrancourt J, Beauvillain C. Le larynx de l'efant. Paris: Arnette Ed; 1979.

9. Hasslinger F. Zur Pathogenese, Diagnostik und Therapeutik des Stridor Congenitus. Z Hals Nasen Ohrenheilkd 1928; 21:223-35.

10. Schwartz L. Congenital Laryngeal Stridor. Arch Otolaryngol 1944; 39:403-12.

11. Lane W, Weider DJ, Steiner C, et al. Laryngomalacia. Arch Otolaryngol 1984;110:546-50.

12. Zarzal GH, Anon JB, Cotton R. Epiglottoplasty for the treatment of laryngomalacia. Ann Otol Rhinol Laryngol 1987;96:72-6.

13. Polonovski JM, Contencin P, François M, Viala P, Narcy P. Aryepiglottic fold excision for the treatment of severe laryngomalacia. Ann Otol Rhinol Laryngol 1990; 99:625-7.

14. Olney DR, Greinwald JH, Smith RJ, Bauman NM. Laryngomalacia and its treatment. Laryngoscope 1999; 109:1770-5.

15. Holinger LD, Konior RJ. Surgical management of severe laryngomalacia. Laryngoscope 1989; 99:136-42.

Endereço para correspondência:

Dr. José Carlos Fraga

Rua Ramiro Barcelos 2350, sala 600 - $6^{\circ}$ andar

CEP 90430-000 - Porto Alegre, RS

Fone: (51) 3316.8232

E-mail: jcfraga@conex.com.br 